\title{
Araştırma Makalesi https://doi.org/10.33484/sinopfbd.701059 Malahit Yeşilinin Şeftali Çekirdeği Kabuğu Üzerine Adsorpsiyon İzotermi ve Termodinamiği
}

\author{
Ali ÇIÇEKÇİ ${ }^{a}$, Bünyamin DÖNMEZ ${ }^{b *}$, Erbil KAVCI ${ }^{a}, \ddot{O} m e r L A C ̧ I ̇ N^{b}$ \\ ${ }^{a}$ Kafkas University, Chemical Engineering Department, Kars-TURKEY \\ ${ }^{\mathrm{b}}$ Ataturk University, Chemical Engineering Department, Erzurum-TURKEY
}

$\ddot{\mathbf{O z}}$

$\mathrm{Bu}$ çalışmada, malahit yeşilinin ham şeftali çekirdeği kabuğu üzerine yaygın adsorpsiyon izoterm modelleri ve termodinamik analizi incelenmiştir. Elde edilen sonuçlardan, korelasyon katsayılarına göre en uygun modelin Freundlich izotermi olduğu belirlenmiştir. Ayrıca, hesaplanan $\mathrm{n}$ değerinin 1'den büyük olması adsorbentin heterojen bir yüzeye sahip olduğunu göstermiştir. Langmuir izoterm modelinde ise, maksimum adsorpsiyon kapasitesi $8.19 \mathrm{mg} \cdot \mathrm{g}^{-1}$ olarak hesaplanmıştır. Ayrıca, termodinamik analizlerden, serbest entalpi değişiminin $-21.53 \mathrm{~kJ} \mathrm{~mol}^{-1}$ ve Gibbs serbest enerji değerinin ise yaklaşı $-39 \mathrm{~kJ} \mathrm{~mol}^{-1}$ olmas1, adsorpsiyon prosesinin ekzotermik, fiziksel karakterli ve kendiliğinden yürüdüğünü ortaya koymaktadır. Sonuç olarak, şeftali çekirdeği kabuğunun, sulu çözeltilerdeki malahit yeşilinin uzaklaştırılması hususunda uygun bir adsorbent olarak kullanılabilir. Ayrıca elde edilen izoterm modeli ve termodinamik parametrelerin, endüstriyel olarak malahit yeşilinin giderilmesi prosesine faydalı veriler sağlanacağı söylenebilir.

Anahtar kelimeler: Şeftali çekirdeği kabuğu, Malahit yeşili, Adsorpsiyon izotermi, Termodinamik parametreler

\section{Adsorption Isotherms and Thermodynamics on Peach Kernel Peel of Malahit Green}

\begin{abstract}
In this study, common adsorption isotherm models and thermodynamic analysis of malachite green on raw peach kernel peel are investigated. From the results obtained, it is determined that the most suitable model according to the correlation coefficients is Freundlich isotherm. In addition, the calculated $\mathrm{n}$ value greater than 1 indicates that the adsorbent has a heterogeneous surface. In Langmuir isotherm model, the maximum adsorption capacity is calculated as $8.19 \mathrm{mg} \cdot \mathrm{g}^{-1}$. Also, from the thermodynamic analysis, the free enthalpy change of $-21.53 \mathrm{~kJ} \cdot \mathrm{mol}^{-1}$ and the Gibbs free energy value of about $-39 \mathrm{~kJ} . \mathrm{mol}^{-1}$ reveal that the adsorption process is exothermic, physically and spontaneous. As a result, the peach kernel skin can be used as a suitable adsorbent for removing malachite green in aqueous solutions. In addition, it can be said that the isotherm model and thermodynamic parameters obtained will provide useful data for the industrial greenhouse removal process.
\end{abstract}

Keywords: Peach kernel peel, Malachite green, Adsorption isotherm, Thermodynamic parameters

\footnotetext{
* Sorumlu Yazar: ORCID ID: orcid.org/ 0000-0002-7680-0755 e-mail: bdonmez@atauni.edu.tr
} 
Giris

Boyar maddeler, kağıt, tekstil, ilaç, deri gibi bir çok sanayi dalında renklendirici olarak kullanımı sonucu atık su olarak çevreyi kirletmektedir. Bu durum, toprak, canlı ve insan sağlığını olumsuz yönde etkilemektedir. Buyüzden atık su içerisindeki boyar madde konsantrasyonunun limit değerlere düşürülmesi gerekmektedir [1-3]. Önemli boyar maddelerden biri olan malahit yeşili, bağışıklık ve üreme sistemine zarar veren genotoksik ve kanserojenik bir maddedir. Çoğu ülkede kullanımı yasaklanmasına rağmen, ucuzluğu ve kolay bulunabilmesinden dolayı özellikle tekstil, yiyecek ve ilaç gibi endüstrilerde sıkça kullanılmaktadır [4,5].

Boyar maddelerin sulardan giderilmesinde kimyasal ve fiziksel olarak birçok yöntem kullanılmaktadır. Adsorpsiyon yöntemi ucuz ve kolay uygulanabilmesi nedeniyle, sulu çözeltilerden boyar madde giderimi konusunda çokça tercih edilmektedir [6-8].

Adsorpsiyon proseslerinde, adsorbat ile adsorbent arasında dinamik bir denge vardır. Denge şartlarında elde edilen bulguların matematiksel bir modelinin geliştirilmesi gerekir. Bunun için elde edilen deneysel bulgular, literatürde bulunan yaygin izoterm modellerine

uygulanır ve sonrasinda en uygun model belirlenir. Böylece bu model, adsorpsiyon mekanizması ve adsorbentin afinitesi hakkında önemli bilgiler sağlar $[9,10]$. Çözeltilerdeki boyar madde veya bir çok kirletici kimyasalın giderilmesinde en çok kullanılan adsorbent, adsorplama kapasitesinin çok yüksek olmasından dolayı aktif karbondur. Fakat bu adsorbent çok pahalı olduğu için literatürde düşük maliyetli ve adsorplama kapasitesi yüksek bir çok adsorbentin kullanılabilirliği araştırılmıştır [11,12]. Bu adsorbentlerden biri de şeftali çekirdeği kabuğudur. Şeftali ağacı gülgiller ailesinden, hoş kokulu bir meyve olup latince ismi Prunus Persia'dır. Dünya'da şeftali üreten ülkeler ve üretim miktarları Tablo 1'de verilmiştir [13]. Ayrıca Türkiye'de şeftali üreten iller ve üretim miktarları Tablo 2'de sunulmuştur [14].

Tablo 1. Dünyada önemli şeftali üreticileri ve miktart

$\begin{array}{lc}\text { Ülke } & \text { Üretim (ton) } \\ \text { Çin } & 12.423 .700 \\ \text { İspanya } & 1.573 .640 \\ \text { İtalya } & 1.379 .428 \\ \text { Yunanistan } & 962.580 \\ \text { ABD } & 959.983 \\ \text { Türkiye } & 608.513 \\ \text { İran } & 575.457\end{array}$


Tablo 2.Türkiye'de şeftali üreten iller ve

\begin{tabular}{|c|c|}
\hline \multicolumn{2}{|c|}{ üretim miktarları } \\
\hline İl & Üretim (ton) \\
\hline Mersin & 103.595 \\
\hline Çanakkale & 91.558 \\
\hline Bursa & 77.941 \\
\hline İzmir & 74.311 \\
\hline Antalya & 17.154 \\
\hline Sakarya & 16.076 \\
\hline Adana & 14.823 \\
\hline
\end{tabular}

Tablolardan görüleceği üzere dünyada ve Türkiye'de önemli miktarlarda şeftali üretimi gerçekleştirilmektedir. Şeftali çekirdeğinin meyve içerisindeki kütlece bileşimi \% 6 civarındadır. Bu sonuç, yıllık çekirdek miktarının ortalama olarak 40.000 ton olması demektir. Bu çekirdek, başta yakıt olmak üzere birkaç farklı alanda kullanılsa bile çoğu zaman bir atık olarak da atılabilmektedir [15]. Dolayısıyla, şeftali meyvesinin bir atığı olan ham şeftali çekirdeğinin oldukça iyi bir potansiyele sahip olduğu ve çok ucuz bir adsorbent olarak kullanılabileceği ön görülmüştür. Bu çalışmada, tehlikeli bir kimyasal atık olan malahit yeşilinin, yüksek potansiyele sahip bir atık olan ham şeftali çekirdeği ile adsorpsiyonuna yönelik izoterm modelleri ve termodinamik analizi amaçlanmıştır.

\section{Materyal-Yöntem}

Merck firmasindan temin edilen malahit yeşili, $\mathrm{C}_{23} \mathrm{H}_{25} \mathrm{~N}_{2} \mathrm{Cl}$ kimyasal formülüne sahiptir (mol kütlesi $=364.92$ g.mol ${ }^{-1}$, C.I. Siniflandirma Numarası 42.000, CAS 569-64-2 $\lambda \max =617 \mathrm{~nm})$. Antalya-Korkuteli'nden temin edilmiş olan ham şeftali çekirdeği kabuğu temizlenip kurutulduktan sonra ögütülmüş ve 600 meşin altına elenmiştir. Bir şeftali çekirdeği kabuğunun elementel analizi Tablo 3' de verilmiştir [15].

Tablo 3. Şeftali çekirdeğinin elementel analizi Şeftali Çekirdeği Bileşimi \% (w/w)

C 50.50

$\mathrm{H}$

$\mathrm{N}_{2}$

0.072 $\mathrm{S}$

$\mathrm{O}$

43.75

Adsorpsiyon denemeleri kesikli sistemde gerçekleştirilmiş olup, öncelikle 1000 ppm'lik malahit yeşili stok çözeltisi hazırlanmış ve tüm deneylerde kullanılan farklı konsatrasyonlardaki çözeltiler için bu stok çözeltisi kullanılmıştır.

\section{Adsorpsiyon Deneyleri}

Adsorpsiyon denemeleri, hava banyolu çalkalayıcı ortamda (Edmund Bühler Gmbh KS-15), 250 mL'lik şilifli erlenler içerisinde gerçekleştirilmiştir. Her bir deneme için 50-100-150-200 ve 250 
ppm boyar madde içeren çözeltilerden 50 $\mathrm{mL}$ alınmış ve 1 gram adsorbent ilave edilerek adsorpsiyon prosesi başlatılmıştır. $\mathrm{Bu}$ işlem denge süresi olan 120 dakikaya kadar devam etmiştir. Deneylerde ortam sıcaklığı $298 \mathrm{~K}$, çalkalama hızı $225 \mathrm{rpm}$ ve serbest $\mathrm{pH}$ değeri 5,2 olarak sabit tutulmuştur. Her bir deney sonunda ortamdan çekilen $5 \mathrm{~mL}$ 'lik numune Nuve NF 1215 modele sahip bir cihazda 5000 rpm 'de santrifüjlenmiştir. Elde edilen nihai çözeltideki boyar madde konsantrasyonu, Mapada V-1100 model spektrofotometre ile tayin edilmiş olup adsorpsiyon kapasitesi Eşitlik 1'den hesaplanmıștır.

$\mathrm{q}_{\mathrm{e}}=\left(\mathrm{C}_{0}-\mathrm{C}_{\mathrm{e}}\right) \frac{\mathrm{v}}{\mathrm{m}}$

Burada $\mathrm{C}_{0}$ ve Ce sırasıyla, başlangıç ve denge anındaki malahit yeşili konsantrasyonlarını (mg.L $\left.{ }^{-1}\right), \quad \mathrm{V}$ çözelti hacmini (L), m adsorbent miktarını (g) ve $\mathrm{q}_{\mathrm{e}}$ ise adsorpsiyon kapasitesini (mg.g ${ }^{-1}$ ) ifade etmektedir.

Brunauer-Emmett-Teller (BET) ve Barrett-Joyner-Halenda (BJH) ile adsorbentin yüzey özellikleri Tablo 4'de verilmiştir. Ayrıca, $\mathrm{p} / \mathrm{p}^{0}$ değeri ise 0.27 olarak bulunmuştur.

Tablo 4. Adsorbentin Yüzey Özellikleri

\begin{tabular}{|l|l|}
\hline Yüzey alanı $\left(\mathrm{S}, \mathrm{m}^{2} \cdot \mathrm{g}^{-1}\right)$ & 17.36 \\
\hline Por Hacmi $\left(\mathrm{Vp}, \mathrm{cm}^{3} \cdot \mathrm{g}^{-1}\right)$ & 0.016 \\
\hline Ortalama Por Boyutu $(\mathrm{dp}, \mathrm{nm})$ & 3.37 \\
\hline
\end{tabular}

\section{Adsorpsiyon İzoterm Modelleri}

Sulu çözeltilerdeki adsorbatın adsorpsiyonuna yönelik, yaygın olarak kullanılan Langmuir, Freundlich, Temkin ve Dubinin-Raduskhevic (D-R) izoterm modelleri incelenmiştir. Yaygın olarak kullanılan izoterm modelleri Tablo 5'de verilmiştir.

\section{Langmuir izotermi}

Langmuir izotermi adsorpsiyonun tek tabakalı olduğunu, adsorpsiyon sitelerinin aynı eş enerjili olduğunu ve adsorplanan madde ile komşu sitelerin birbirini etkilemediğini kabul eder. Önemli bir karakteristik özelliği ise $R_{L}$ değeridir. $R_{L}$ değeri boyutsuz olup adsorpsiyonun tipini belirler. Eğer $R_{L}>1$ ise adsorpsiyon elverişsiz, $R_{L}=1$ ise lineer, $R_{L}=0$ ise geri dönüşümsüz, $0<\mathrm{R}_{\mathrm{L}}<1$ ise elverişli olduğunu belirtir.

\section{Freundlich izotermi}

Freundlich izotermi heterojen sistemler için tanımlanan çok tabakalı adsorpsiyon modeldir. $\mathrm{Bu}$ modelde adsorpsiyon 1sısının dağglımı üniform değildir ve yüzey heterojendir. Burada heterojenlik faktörü $\mathrm{n}>1$ ise adsorpsiyon elverişlidir [16].

\section{Temkin izotermi}

Temkin adsorpsiyon izotermi, adsorbent-adsorbat etkileşimlerinin 
adsorpsiyon üzerindeki etkilerini ifade eden bir adsorpsiyon izotermidir. $\mathrm{Bu}$ izoterme göre, tabakadaki bütün moleküllerin adsorpsiyon 1sısı lineer olarak azalmaktadır. yanı sıra heterojen bir yüzeye olan görünür adsorpsiyon enerjilerini (özellikle gözenekli adsorbentlerde) tahmin etmek için test edilir.

\section{Dubinin-Radushkevich izotermi}

D-R izotermi, çoğunlukla

adsorbentin karakteristik gözenekliliğinin

Tablo 5. Kullanılan izotermler ve matematiksel ifadeleri

\section{İzoterm türü}

Langmuir

Freundlich

Temkin

Dubinin-Radushkevich

\section{İzoterm modeli}

$$
\frac{\mathrm{C}_{\mathrm{e}}}{\mathrm{q}_{\mathrm{e}}}=\frac{\mathrm{C}_{\mathrm{e}}}{\mathrm{q}_{\mathrm{m}}}+\frac{1}{\mathrm{~K}_{\mathrm{L}} \times \mathrm{q}_{\mathrm{m}}}, \mathrm{R}_{\mathrm{L}}=\frac{1}{1+\mathrm{K}_{\mathrm{L}} \mathrm{C}_{0}}
$$

$$
\ln \left(\mathrm{q}_{\mathrm{e}}\right)=\ln \left(\mathrm{K}_{\mathrm{f}}\right)+\frac{1}{\mathrm{n}} \times \ln \left(\mathrm{C}_{\mathrm{e}}\right)
$$

$$
\mathrm{q}_{\mathrm{e}}=\mathrm{B}_{\mathrm{T}} \times \ln \left(\mathrm{K}_{\mathrm{T}}\right)+\mathrm{B}_{\mathrm{T}} \times \ln \left(\mathrm{C}_{\mathrm{e}}\right)
$$

$$
\ln \left(\mathrm{q}_{\mathrm{e}}\right)=\ln \left(\mathrm{q}_{\mathrm{m}}\right)+\beta \times \varepsilon^{2}
$$

$$
\varepsilon=\mathrm{R}_{\mathrm{g}} \operatorname{Tln}\left(1+\frac{1}{\mathrm{C}_{\mathrm{e}}}\right)
$$

\section{Referans}

[20]

\section{Bulgular ve Tartışma}

Malahit yeşilinin şeftali çekirdeği kabuğu üzerine olan adsorpsiyon çalışmasında, en uygun izoterm modelini belirlemek için Tablo 5'deki eşitlikler kullanılmıştır. Eşitlik 2'den, $C_{e}$ ye karş1 $\mathrm{C}_{\mathrm{e}} / \mathrm{q}_{\mathrm{e}}$ değerleri grafik edilerek Langmuir

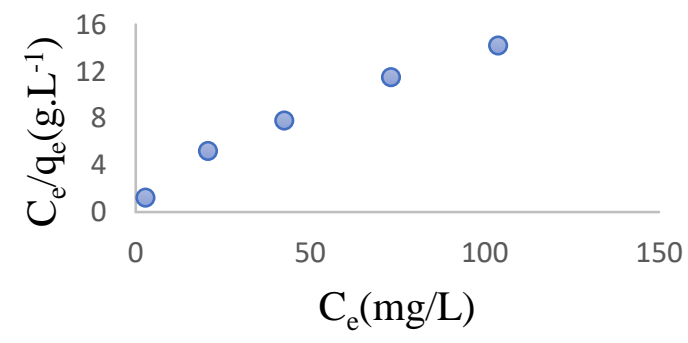

Şekil 1. Langmuir izotermi sabitleri bulunmuştur. Diğer izoterm sabitleri de aynı yol takip edilerek belirlenmiştir (Şekil 1-4). Ayrıca, bu izotermlerden hesaplanan sabitler ve korelasyon katsayıları Tablo 6'da verilmiştir.

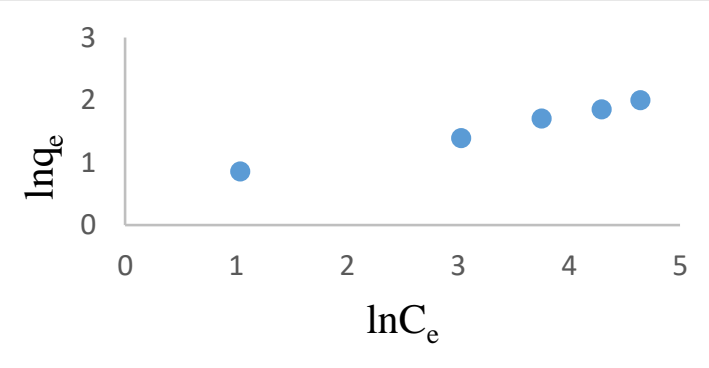

Şekil 2. Freundlich İzotermi 


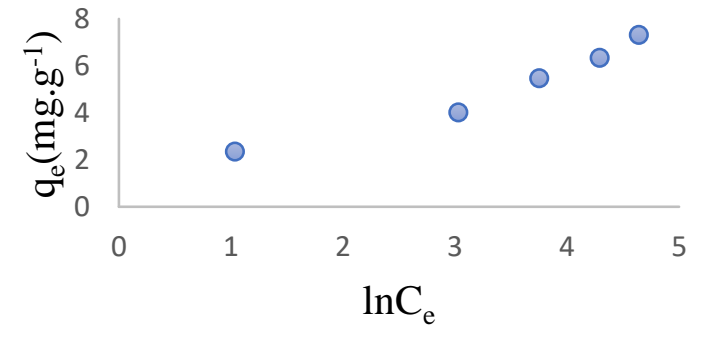

Şekil 3. Temkin İzotermi

Tablo 6. İzoterm Parametreleri ve $R^{2}$ Değerleri

İzoterm parametresi

Değeri

\section{Langmuir}

$\begin{array}{ll}\mathrm{q}_{\mathrm{m}}\left(\mathrm{mg} \cdot \mathrm{g}^{-1}\right) & 8.19 \\ \mathrm{~K}_{\mathrm{L}}\left(\mathrm{L} \cdot \mathrm{g}^{-1}\right) & 0.065 \\ \mathrm{R}_{\mathrm{L}} & 0.133 \\ \mathrm{R}^{2} & 0.97\end{array}$

Freundlich

$\begin{array}{ll}\mathrm{K}_{\mathrm{f}}\left[\left(\mathrm{mg} \cdot \mathrm{g}^{-1}\right)\left(\left(\mathrm{L} \cdot \mathrm{g}^{-1}\right)^{-1} / \mathrm{n}\right]\right. & 1.66 \\ \mathrm{n} & 3.19 \\ \mathrm{R}^{2} & 0.99\end{array}$

Temkin
$\mathrm{K}_{\mathrm{T}}\left(\mathrm{L} \cdot \mathrm{g}^{-1}\right)$
1.6
$\mathrm{B}_{\mathrm{T}}\left(\mathrm{kJ} \cdot \mathrm{mol}^{-1}\right)$
1.332
$\mathrm{R}^{2}$

Dubinin-Radushkevich

$\begin{array}{ll}\beta\left(\mathrm{mol}^{2} \cdot \mathrm{kJ}^{-2}\right) & 0.0004 \\ \mathrm{q}_{\mathrm{m}}\left(\mathrm{mg} \cdot \mathrm{g}^{-1}\right) & 1.9633 \\ \mathrm{R}^{2} & 0.93\end{array}$

Tablo 6 incelendiğinde Langmuir izotermine göre $\mathrm{R}^{2}=0.97$ ve $\mathrm{R}_{\mathrm{L}}=0.133$ olarak bulunmuştur. $\mathrm{R}_{\mathrm{L}}$ değerinin 0 ila 1 arasında olması, kullanılan adsorbentin

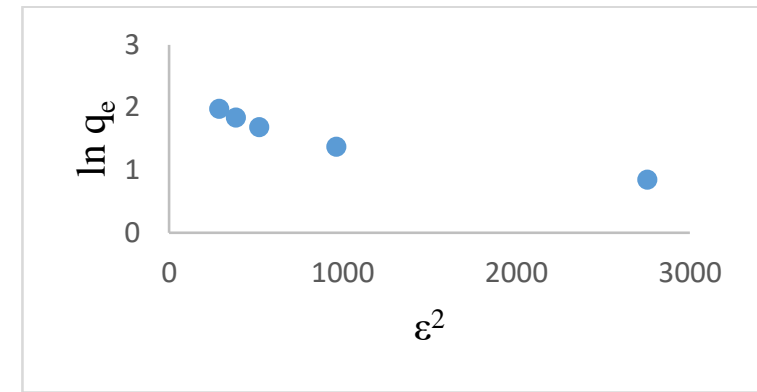

Şekil 4. Dubinin-Radushkevich izoterm

malahit yeşilini iyi bir şekilde adsorplayabileceğini göstermektedir. Freundlich izoterminde $\mathrm{R}^{2}$ değerinin 0.99 olarak bulunması ise adsorpsiyon prosesinin bu modelle oldukça iyi bir uyum içerisinde olduğunu ortaya koymaktadır. $\mathrm{Bu}$ modelde $\mathrm{K}_{\mathrm{f}}$ değeri 1.66 olarak bulunmuş ve n değerinin 1 ila 10 arasında olması ise, adsorbentin heterojen bir yüzeye sahip olduğunu ve adsorplama yeteneğinin iyi olduğunu ifade etmektedir. Temkin izoterminde, $\mathrm{B}_{\mathrm{T}}$ değerinin $1.332 \mathrm{~kJ} \cdot \mathrm{mol}^{-1}$ olarak bulunması, adsorpsiyonun fiziksel olabileceğini göstermektedir. İlaveten, D-R izotermine ait $\mathrm{R}^{2}$ değeri 0.93 olması, diğer izotermlere göre daha az uyum içerisinde olduğunu göstermiştir.

\section{Adsorpsiyon termodinamiği}

Termodinamik parametreler olarak kullanılan Gibbs serbest enerji değişimi $\left(\Delta \mathrm{G}^{\mathrm{o}}\right)$, entalpi değişimi $\left(\Delta \mathrm{H}^{\circ}\right)$ ve entropi değişimi $\left(\Delta \mathrm{S}^{\mathrm{o}}\right)$, adsorpsiyon prosesinin doğasını açıklamak için aşağıdaki eşitliklerden hesaplanır (Eşitlik 6-8). 
$\mathrm{K}_{\mathrm{c}}=\frac{\mathrm{q}_{\mathrm{e}}}{\mathrm{C}_{\mathrm{e}}}$

$\ln \left(\mathrm{K}_{\mathrm{c})}=\left(\Delta \mathrm{S}^{\circ} / \mathrm{R}_{\mathrm{g}}\right)-\left(\Delta \mathrm{H}^{\circ} / \mathrm{R}_{\mathrm{g}} \mathrm{T}\right)\right.$

$\Delta \mathrm{G}^{\circ}=-\mathrm{R}_{\mathrm{g}} \mathrm{T} \times \ln \left(\mathrm{K}_{\mathrm{c}}\right)=\Delta \mathrm{H}^{\circ}-\mathrm{T} \Delta \mathrm{S}^{\circ}$

$\Delta \mathrm{H}^{\mathrm{o}}$ değeri, adsorpsiyon işleminin fiziksel $\left(80<\mathrm{kJ} . \mathrm{mol}^{-1}\right)$ veya kimyasal $(80-$ $200 \quad \mathrm{~kJ} . \mathrm{mol}^{-1}$ ) olup olmadı̆̆ını belirlemektedir [20].
Adsorpsiyon termodinamiği için beș farklı sicaklıkta $(298,303,308,313,318 \mathrm{~K})$ denemeler yapılmıştır. Deneysel verilere göre, Eşitlik 6-8 ve Şekil 5'in kullanılmasıyla, $\quad \Delta \mathrm{H}^{\mathrm{o}}, \quad \Delta \mathrm{S}^{\mathrm{o}} \quad$ ve $\quad \Delta \mathrm{G}^{\mathrm{o}}$ parametreleri hesaplanmıştır. Hesaplanan bu parametreler Tablo 7' de verilmiştir.

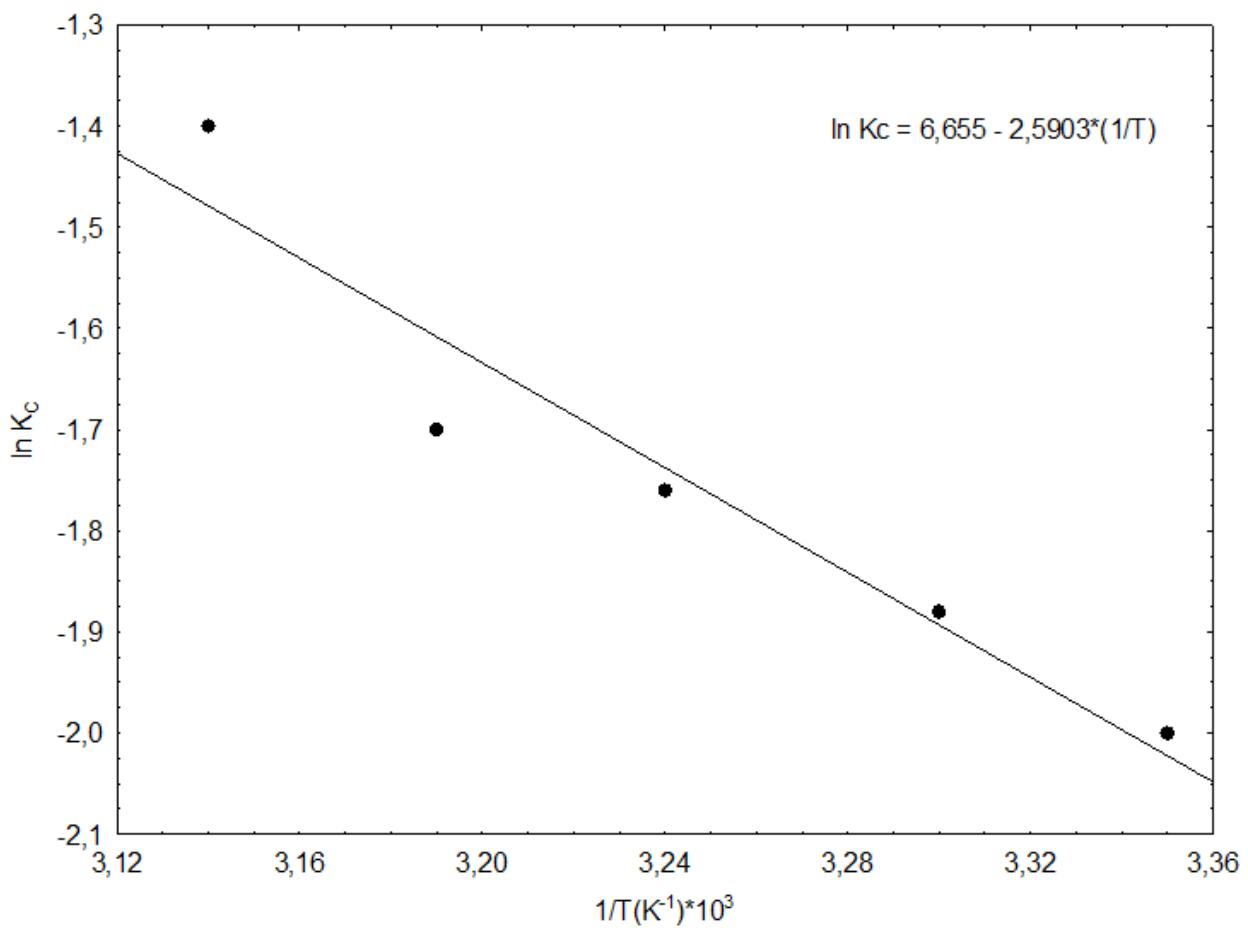

Şekil 5. In $K_{C}-1 /$ T grafiği

Tablo 7. Adsorpsiyon prosesi için termodinamik parametreler

$\Delta \mathrm{H}^{\circ}\left(\mathrm{kJ} \cdot \mathrm{mol}^{-1}\right) \quad \Delta \mathrm{S}^{\circ}\left(\mathrm{J} \cdot \mathrm{mol}^{-1} \cdot \mathrm{K}^{-1}\right) \quad \Delta \mathrm{G}^{\circ}\left(\mathrm{kJ} \cdot \mathrm{mol}^{-1}\right)$

\begin{tabular}{lllllll}
\hline & & $298 \mathrm{~K}$ & $303 \mathrm{~K}$ & $308 \mathrm{~K}$ & $313 \mathrm{~K}$ & $318 \mathrm{~K}$ \\
-21.53 & 55.4 & -38.03 & -38.30 & -38.60 & -38.90 & -39.10 \\
\hline
\end{tabular}


Tablo 7'de verilen değerler incelendiğinde, $\Delta \mathrm{H}^{\circ}$ '1n negatif olması adsorpsiyonun ekzotermik olduğunu, $\Delta S^{\circ}$ 'in pozitif olması ise adsorbent - adsorbat arayüzeyinde düzensizliğin az da olsa arttığını göstermektedir. Ayrıca, $\Delta G^{\circ}$ değerinin negatif çıkması adsorpsiyon prosesin kendiliğinden yürüdüğünü ortaya koymaktadır.

\section{Sonuçlar}

Malahit yeşilinin ham şeftali çekirdek kabuğu üzerine olan adsopsiyonunda, farklı izoterm modelleri incelenmiş ve adsorpsiyon termodinamiği üzerine analizler yapılarak ilgili parametreler değerlendirilmiştir. Yapılan çalışmalar sonucunda, Freundlich izoterm modelinin en uygun model olduğu gözlenmiştir $\left(\mathrm{R}^{2}=0.99\right)$. Ayrıca, hesaplanan n değeri 1'den büyük çıkmıştır, bu ise, adsorbentin heterojen bir yüzeye sahip olduğunu ve belli düzeyde bir adsorplama kapasitesine sahip olduğunu göstermiştir. Termodinamik analizlerden, $\Delta \mathrm{H}^{\circ}$ değeri 21.53 kj. $\mathrm{mol}^{-1}$ olarak hesaplanmış, ki bu durum, adsorpsiyon prosesinin ekzotermik ve aynı zamanda fiziksel olduğunu desteklemiştir. Ayrıca, $\Delta \mathrm{G}^{\circ}$ değerinin negatif olması ise, adsorpsiyonun kendiliğinden yürüdüğünü göstermektedir. Bütün bu bulgular 1şığında, malahit yeşilinin adsorpsiyonu için ülkemizde önemli bir atık potansiyeline sahip olan şeftali çekirdeğinin uygun bir adsorbent olabileceği düşünülmektedir.

\section{Kaynaklar}

[1] Garg V. K, Gupta R, Bala Yadav A, Kumar R, 2003. Dye removal from aqueous solution by adsorption on treated sawdust. Bioresource Technology, 89 (2): 121-124.

[2] Erkmen J, 2018. The use of hydroxyethyl cellulose as a transparent filling material in finishing polish. Pigment \& Resin Technology, 47(4): 323-329.

[3] Kupeta AJK, Naidoo EB, Ofomaja AE, 2018. Kinetics and equilibrium study of 2-nitrophenol adsorption onto polyurethane cross-linked pine cone biomass. Journal of Cleaner Production, 179: 191-209.

[4] Srivastava S, Sinha R, Roy D, 2004. Toxicological effects of malachite green. Aquatic Toxicology, 66(3): 319-329.

[5] Santhi T, Manonmani S, 2011. Malachite Green Removal from Aqueous Solution by the Peel of Cucumis sativa Fruit. CLEAN - Soil, Air, Water, 39(2):162-170.

[6] Subbaiah MV, Kim DS, 2016. Adsorption of methyl orange from aqueous solution by aminated pumpkin seed powder: Kinetics, isotherms, and thermodynamic studies. Ecotoxicology and Environmental Safety, 128:109-117.

[7] Lacin O, Haghighatnia A, Demir F, Sevim F, 2019. Adsorption Characteristics and Behaviors of Natural Red Clay for Removal of BY28 from Aqueous Solutions. International Journal of Trend in Scientific 
Research and Development, 3(2):10371047.

[8] Adeyemo AA, Adeoye IO, Bello OS, 2017. Adsorption of dyes using different types of clay: a review. Appl Water Sci, 7(2):543-568.

[9] Sen TK, Afroze S, Ang HM, 2011. Equilibrium, Kinetics and Mechanism of Removal of Methylene Blue from Aqueous Solution by Adsorption onto Pine Cone Biomass of Pinus radiata. Water Air Soil Pollut, 218(1): 499-515.

[10] Foo KY, Hameed BH, 2010. Insights into the modeling of adsorption isotherm systems. Chemical Engineering Journal, 156(1): 2-10.

[11] Banerjee S, Chattopadhyaya MC, 2017. Adsorption characteristics for the removal of a toxic dye, tartrazine from aqueous solutions by a low cost agricultural by-product. Arabian Journal of Chemistry 10(2): S1629-S1638.

[12] Rabia K, Özer Ç, 2018. Remazol Brillant Blue R (RBBR) boyarmaddesinin düşük maliyetli bir adsorban olan sigara külü ile giderimi. Sakarya University Journal of Science, 22(2): 174-180.

[13] Food and Agriculture Organization of the United Nations, 2014. E-learning course: Gender in Food and Nutrition Security and Policy and Legislation. Gender Statistics for Informing Policy and Legislation.

[14] Türkiye İstatistik Kurumu Faaliyet raporu, 2011 , https://www.tuik.gov.tr/jsp/duyuru/upload/ FR-2011.pdf.

[15] Küçük İ, 2019. Farklı biyokütlelerden karbondioksit kullanılarak karbon moleküler elek özelliğinde aktif karbon üretimi ve uygulamaları. Doktora Tezi, İnönü Üniversitesi Fen Bilimleri Enstitüsü, İzmir.

[16] Kalpaklı Y, Toygun Ş, Köneçoğlu G, Akgün M, 2014. Equilibrium and kinetic study on the adsorption of basic dye (BY28) onto raw $\mathrm{Ca}$-bentonite. Desalination and Water Treatment, 52(37-39): 7389-7399.

[17] Langmuir I, 1918. The adsorption of gases on plane surfaces of glass, mica and platinum. JACS. 40: 1361-1403.

[18] Freundlich H, 1906. Over the adsorption in solution. J. Phys. Chem. 57: 1100-1107.

[19] Temkin MI, 1941. Adsorption equilibrium and the kinetics of processes on nonhomogeneous surfaces and in the interaction between adsorbed molecules. Zh. Fiz. Chim.15: 296-332.

[20] Dubinin MM, Radushkevich, LV, 1947. The equation of the characteristic curve of the activated charcoal. Proceedings of the Academy of Sciences, Physical Chemistry Section, 55: 331-337. 